\title{
MULTI-SENSOR PHD BY SPACE PARTITIONING: COMPUTATION OF A TRUE REFERENCE DENSITY WITHIN THE PHD FRAMEWORK
}

\author{
E. DELANDE, E. DUFLOS, P. VANHEEGHE \\ LAGIS FRE CNRS 3303 \\ Ecole Centrale de Lille \\ 59651 Villeneuve d'Ascq, France
}

\author{
D. HEURGUIER
}

\author{
OPS / HAT / SPM \\ Thales Communication \\ 92704 Colombes, France
}

\begin{abstract}
In a previous paper, the authors proposed an extension of the Probability Hypothesis Density (PHD), a well-known method for singlesensor multi-target tracking problems in a Bayesian framework, to the multi-sensor case. The true expression of the multi-sensor data update PHD equation was constructed using finite sets statistics (FISST) derivative techniques on functionals defined on multi-sensor observation and state space named "cross-terms". In this paper, an equivalent expression in a combinational form is provided, which allows an easier interpretation of the data update equation. Then, using the joint partitioning proposed by the authors in the previous paper, an exact multi-sensor multi-target PHD filter is efficiently propagated on a benchmark scenario involving 10 sensors and up to 10 simultaneous targets where the brute force approach would have been extremely burdensome. The availability of a true reference PHD then allows a validation of the classical iterated-corrector approximation method, albeit limited to the scope of the implemented scenario.
\end{abstract}

Index Terms - Probability Hypothesis Density, Multi-sensor system, Multi-target tracking

\section{INTRODUCTION}

In the general multi-sensor multi-target Bayesian framework, an unknown (and possibly varying) number of targets whose states $x_{1}, \ldots x_{n}{ }^{1}$ are observed by several sensors which produce a collection of measurements $z_{1}, \ldots, z_{m}$ at every time step $k$. Mahler's work on FISST ([1]) provides a mathematical framework to build multi-object densities and derive the Bayesian rules. Randomness on object number and their states are encapsulated into random finite sets (RFS), namely multi-target (state) sets $X=\left\{x_{1}, \ldots, x_{n}\right\}$ and multi-sensor (measurement) set $Z_{k}=\left\{z_{1}, \ldots, z_{m}\right\}$. The objective is then to propagate the multi-target probability density $f_{k \mid k}\left(X \mid Z^{(k)}\right)$ by using the Bayesian set equations at every time step $k$ :

$$
\begin{aligned}
f_{k+1 \mid k}\left(X \mid Z^{(k)}\right) & =\int f_{k+1 \mid k}(X \mid W) f_{k \mid k}\left(W \mid Z^{(k)}\right) \delta W \\
f_{k+1 \mid k+1}\left(X \mid Z^{(k+1)}\right) & =\frac{f_{k+1}\left(Z_{k+1} \mid X\right) f_{k+1 \mid k}\left(X \mid Z^{(k)}\right)}{\int f_{k+1}\left(Z_{k+1} \mid W\right) f_{k+1 \mid k}\left(W \mid Z^{(k)}\right) \delta W}
\end{aligned}
$$

This work was supported by the Direction Générale de l'Armement (DGA) and the Centre National de la Recherche Scientifique (CNRS)

${ }^{1}$ The target state $x_{i} \in \mathcal{X}$ is usually composed of a position, a velocity, where $Z^{(k)}=\bigcup_{t \leqslant k} Z_{t}$ is the collection of measurements up to time $k, f_{k \mid k}\left(W \mid Z^{(k)}\right)$ is the current multi-target posterior density in set $W, f_{k+1 \mid k}(X \mid W)$ is the current multi-target Markov transition density, from set $W$ to set $X, f_{k+1}(Z \mid X)$ is the current multi-sensor multi-target likelihood function.

Even though equations (1), (2) are well built within the FISST framework, they are untractable because of the set integrals and the probability densities defined on multi-object spaces. Mahler proposed in [2] to limit the propagation of the multi-target probability density $f_{k \mid k}\left(X \mid Z^{(k)}\right)$ to its first-moment density, the PHD $D_{k \mid k}\left(x \mid Z^{(k)}\right)$. The PHD encapsulates information on both target number and states but, being defined on the single-state space $\mathcal{X}$, its propagation does not require the computation of cumbersome set integrals or multiobject densities. Under certain assumptions on the target motion and the observation models, Mahler provided in [2] the tractable PHDequivalents of Bayesian set equations (1) and (2), the latter in the single-sensor case only.

In a previous paper ([3]), the authors extended Mahler's work and provided a true multi-sensor data update equation in a derivative form. Here, an equivalent expression in a combinational form is given; it allows an intuitive intepretation of the data update equation and made easier the comparison with Mahler's own extension to the two-sensor case ([4]), which turned to be conclusive. This paper also provides simulation results from the comparison between the PHD propagated by the true data update equation and by the classical iterated-corrector approximation on a given scenario. Note that the theoretical results presented here are more detailed in [5], and that a full understanding of this work requires some knowledge about FISST theory and calculus rules which may be found in [1].

\section{MULTI-SENSOR DATA UPDATE EQUATION}

Following the time update step and with the same assumption than exposed by Mahler ([2]), the updated distribution $f_{k+1 \mid k}\left(X \mid Z^{(k)}\right)$ is assumed Poisson with parameter $\mu$ and intensity $\mu s(x)^{2}$. Since $f_{k+1 \mid k}\left(X \mid Z^{(k)}\right)$ is Poisson, its intensity $\mu s(x)$ equals the time updated PHD $D_{k+1 \mid k}\left(x \mid Z^{(k)}\right)$ ([2]). Note that the following notations were chosen as close as possible to Mahler's work for clarity's sake. \footnotetext{
sake

${ }^{2} \mu s()=.\mu_{k+1 \mid k} s_{k+1 \mid k}($.$) , time subscripts are omitted for simplicity's$
} 


\subsection{Observation model}

Assume that, following target transition between time steps $k$ and $k+1$, each sensor $j \in[1 N]$ produces measurements independently of the others according to the observation model described as follows:

- Target $i$ is detected with probability $p_{d}^{[j]}\left(x_{k+1}^{i}\right)^{3}$;

- If detected, target $i$ produces a single measurement $z \in \mathcal{Z}^{[j]}$ with probability distribution $f_{k+1}^{O,[j]}\left(z \mid x_{k+1}^{i}\right)=L_{z}^{[j]}\left(x_{k+1}^{i}\right)$;

- False alarms are Poisson with parameter $\lambda^{[j]}$ and intensity $\lambda^{[j]} c^{[j]}(z)$;

- Observation processes on each target are independent conditionally on the multi-target set $X_{k+1}$.

\subsection{Cross-terms}

Generalizing the single-sensor case led the authors to the introduction and the definition of the cross-terms ([5]) which played an important role in the construction of the multi-sensor data update equation:

Definition 2.1. For each sensor $j \in[1 N]$, let $g^{[j]}$ be a realvalued function on observation space $\mathcal{Z}^{[j]}$ such that $\forall z \in \mathcal{Z}^{[j]}$, $0 \leqslant g^{[j]}(z) \leqslant 1$. Let $h$ be a real-valued function on state space $\mathcal{X}$ such that $\forall x \in \mathcal{X}, 0 \leqslant h(x) \leqslant 1$. The cross-term $\beta\left[g^{[1]}, \ldots, g^{[N]}, h\right]$ is the functional defined by:

$$
\begin{aligned}
\beta\left[g^{[1]}, \ldots, g^{[N]}, h\right] & =\sum_{j=1}^{N}\left(\lambda^{[j]} c^{[j]}\left[g^{[j]}\right]-\lambda^{[j]}\right) \\
& +\mu s\left[h \prod_{j=1}^{N}\left(1-p_{d}^{[j]}+p_{d}^{[j]} p_{g^{[j]}}^{O,[j]}\right)\right]-\mu
\end{aligned}
$$

where $p_{g^{[j]}}^{O,[j]}(x)=\int g^{[j]}(z) f_{k+1}^{O,[j]}(z \mid x) d z$,

$c^{[j]}\left[g^{[j]}\right]=\int g^{[j]}(z) c^{[j]}(z) d z$ and $s[h]=\int h(x) s(x) d x$.

Using FISST calculus rules ([2]), the cross-term $\beta$ can be differentiated on a single-target space point $x \in \mathcal{X}$ and/or an tuple of various single-sensor observation points $z^{[j]} \in \mathcal{Z}^{[j]}$ ([5]). The analytical expressions of the differentiated cross-terms allows an intuitive interpretation as "likelihoods" ${ }^{4}$; for example, with $N=3$ :

- $\left[\frac{\delta \beta\left[g^{[1]}, g^{\left.[2], g^{[3]}, h\right]}\right.}{\delta x}\right]_{g^{[i]}=0, h=1}=\mu s(x) \prod_{j=1}^{3}\left(1-p_{d}^{[j]}(x)\right): \mathrm{a}$ target is in state $x$ and was not detected by any sensor;

- $\left[\frac{\delta^{3} \beta\left[g^{[1]}, g^{[2]}, g^{[3]}, h\right]}{\delta x \delta z^{[1]} \delta z^{[2]}}\right]_{g^{[i]}=0, h=1}=$ $\mu s(x) \prod_{j=1}^{2}\left(p_{d}^{[j]}(x) L_{z^{[j]}}^{[j]}(x)\right)\left(1-p_{d}^{[3]}(x)\right):$ a target is in state $x$, generated measurements $z^{[1]}$ and $z^{[2]}$ and was not detected by sensor 3 ;

- $\left[\frac{\delta^{2} \beta\left[g^{[1]}, g^{[2]}, g^{[3]}, h\right]}{\delta z^{[1]} \delta z^{[2]}}\right]_{g^{[i]}=0, h=1}=$ $\mu s\left[\prod_{j=1}^{2}\left(p_{d}^{[j]} L_{z^{[j]}}^{[j]}\right)\left(1-p_{d}^{[3]}\right)\right]:$ a single target generated measurements $z^{[1]}$ and $z^{[2]}$ and was not detected by sensor 3 .

${ }^{3} p_{d}^{[j]}()=.p_{d, k+1}^{[j]}($.$) , time subscripts are omitted for simplicity's sake$

4 "Likelihood" should not be interpreted in its classical Bayesian sense
Each cross-term above denotes a "likelihood" of a "link" between points in the single-state space $\mathcal{X}$ and/or the observation spaces $\mathcal{Z}^{[j]}$, hence their name.

\subsection{Data update equation}

Denote by $Z_{k+1}^{[j]}=\left\{z_{1}^{[j]}, \ldots, z_{m[j]}^{[j]}\right\}$ the set of current measurements produced by the $j$-th sensor, and by $Z_{k+1}=\left(Z_{k+1}^{[1]}, \ldots, Z_{k+1}^{[N]}\right)$ the current multi-sensor measurement set. The authors proposed in [3] a multi-sensor data update equation constructed as a set differentiation of the cross-term $\beta\left[g^{[1]}, \ldots, g^{[N]}, h\right]$ :

$$
\begin{aligned}
& D_{k+1 \mid k+1}\left(x \mid Z^{(k+1)}\right)= \\
& \frac{\left[\frac{\delta}{\delta x}\left(\frac{\delta^{m[1]+\ldots+m[N]}}{\delta z_{1}^{[1]} \ldots \delta z_{m[N]}^{[N]}} e^{\beta\left[g^{[1]}, \ldots, g^{[N]}, h\right]}\right)\right]_{g^{[i]}=0, h=1}}{\left[\frac{\delta^{m[1]+\ldots+m[N]}}{\delta z_{1}^{[1]} \ldots \delta z_{m[N]}^{[N]}} e^{\beta\left[g^{[1]}, \ldots, g^{[N]}, h\right]}\right]_{g^{[i]}=0, h=1}}
\end{aligned}
$$

Since a single target, if detected by sensor $j$ at the current time step, cannot generate more than one measurement in $\mathcal{Z}^{[j]}$, one can expect $\beta$ to vanish if differentiated in at least two points from the same observation space $\mathcal{Z}^{[j]}$; this is indeed the case within the FISST calculus rules. That is, remaining cross-terms in (4) are differentiated in measurements $z^{\left[k_{1}\right]}, \ldots, z^{\left[k_{n}\right]}$ from different sensors $k_{1}, \ldots, k_{n}$ or, equivalently, on a tuple $\left(z^{\left[k_{1}\right]}, \ldots, z^{\left[k_{n}\right]}\right)$ defined on the cartesian product $\mathcal{Z}^{\left[k_{1}\right]} \times \ldots \times \mathcal{Z}^{\left[k_{n}\right]}$.

Thus, if we denote by:

- $\check{Z}_{N}$ the set of (unordered) $q$-tuples, $1 \leqslant q \leqslant N$, defined on current measurement set $Z_{k+1}$, with at most one measurement from each observation space;

- For any tuple $T_{i}=\left(z^{\left[k_{1}\right]}, \ldots, z^{\left[k_{n}\right]}\right) \in \check{Z}_{N}, \beta\left[T_{i}, h\right]$ the cross-term $\left[\frac{\delta^{n}}{\delta z^{\left[k_{1}\right]} \ldots \delta z^{\left[k_{n}\right]}} \beta\left[g^{[1]}, \ldots, g^{[N]}, h\right]\right]_{g^{[i]}=0}$.

then (4) can be expanded as follows (see [5] for details):

$$
\beta\left[\begin{array}{l}
D_{k+1 \mid k+1}\left(x \mid Z^{(k+1)}\right)= \\
\sum_{T \in \mathcal{T}\left(\check{Z}_{N}\right)} \sum_{T_{i} \in T}\left(\beta\left[T_{i}, \delta_{x}\right] \prod_{\substack{T_{j} \in T \\
T_{j} \neq T_{i}}} \beta\left[T_{j}, 1\right]\right) \\
\sum_{T \in \mathcal{T}\left(\check{Z}_{N}\right)} \prod_{T_{i} \in T} \beta\left[T_{i}, 1\right]
\end{array}\right.
$$

where:

- $T \in \mathcal{P}\left(\check{Z}_{N}\right)$ is a combinational term, i.e. a set of tuples containing each measurement in $Z_{k+1}$ once and only once;

- $\mathcal{T}\left(\check{Z}_{N}\right) \subseteq \mathcal{P}\left(\check{Z}_{N}\right)$ is the set of all combinational terms.

Note that equations (4) and (5) are different forms from the same multi-sensor data update equation and therefore are equivalent. The combinational form (5) provides an easier interpretation of the data update process since, similarly to the cross-terms, each combinational term can be interpreted intuitively as a "likelihood" linking the whole measurement set $Z_{k+1}$ to the state space $\mathcal{X}$.

For example, with $N=2$ and $Z_{k+1}=\left\{z_{1}^{[1]}, z_{2}^{[1]}, z_{1}^{[2]}\right\}$, one of the combinational terms is $\left\{\left(z_{1}^{[1]}, z_{1}^{[2]}\right),\left(z_{2}^{[1]}\right)\right\}$ and it appears in (5) through the following products: 
- $\beta\left[\left(z_{1}^{[1]}, z_{1}^{[2]}\right), 1\right] \beta\left[\left(z_{2}^{[1]}\right), 1\right]:$ a target generated both $z_{1}^{[1]}$ and $z_{1}^{[2]}$, another source generated $z_{2}^{[1]}$ only (either target or false alarm);

- $\beta\left[\left(z_{1}^{[1]}, z_{1}^{[2]}\right), \delta_{x}\right] \beta\left[\left(z_{2}^{[1]}\right), 1\right]$ : a target is in $x$ and generated both $z_{1}^{[1]}$ and $z_{1}^{[2]}$, another source generated $z_{2}^{[1]}$ only (either target or false alarm);

- $\beta\left[\left(z_{1}^{[1]}, z_{1}^{[2]}\right), 1\right] \beta\left[\left(z_{2}^{[1]}\right), \delta_{x}\right]$ : a target is in $x$, generated $z_{2}^{[1]}$ and was not detected by sensor 2 , another target generated both $z_{1}^{[1]}$ and $z_{1}^{[2]}$.

\subsection{Simplification by state and sensor partitioning}

In [5] the authors showed that if the sensor FOVs do not all overlap with each other, many differentiated cross-terms are likely to vanish in the multi-sensor data update (5). That is why the joint partitioning of the sensors and the state space was proposed ([5], [3]):

Definition 2.2. For any sensor $j \in[1 N]$, let $F_{k+1}^{[j]} \subset \mathcal{X}$ denote its field of view at time $k+1$ defined as:

$$
\forall x \in \mathcal{X}, x \in F_{k+1}^{[j]} \Leftrightarrow p_{d, k+1}^{[j]}(x) \neq 0
$$

Define the equivalence relation "cross" $(\leftrightarrow)$ between sensors as:

$$
\forall i, j \in[1 N],(i \leftrightarrow j) \Leftrightarrow\left(F_{k+1}^{[i]} \cap F_{k+1}^{[j]} \neq \emptyset\right)
$$

Let $\left\{P_{S}(p)\right\}_{p=1}^{P}$ be the sensor partition of $[1 N]$ formed by the equivalence classes of the transitive closure of the "cross" relation. Let $\left\{P_{T}(p)\right\}_{p=0}^{P}{ }^{5}$ be the space partition of the state space $\mathcal{X}$ defined by:

$$
P_{T}(p)= \begin{cases}\overline{\bigcup_{j=1}^{N} F_{k+1}^{[j]}} & (p=0) \\ \bigcup_{j \in P_{S}(p)} F_{k+1}^{[j]} & (p \neq 0)\end{cases}
$$

Finally, for any element $P_{S}(p)$ ot the sensor partition, let $n_{p}=$ $\left|P_{S}(p)\right|$ denotes the number of sensors in $P_{S}(p)$, and let $p_{1}, \ldots, p_{n_{p}}$ denote the increasing indexes in $\left[\begin{array}{ll}1 & N\end{array}\right]$ of sensors belonging to $P_{S}(p)$.

Then, equation (5) can be simplified ([5]) and is equivalent to:

$$
\left\{\begin{array}{r}
D_{k+1 \mid k+1}\left(x \mid Z^{(k+1)}\right)= \\
D_{k+1 \mid k}\left(x \mid Z^{(k)}\right) \\
\sum_{p}\left[\emptyset, \delta_{x}\right]+\frac{\sum_{T \in \mathcal{T}\left(\check{Z}_{N p}^{(p)}\right)} \sum_{T_{i} \in T}\left(x \in P_{T}(0)\right)}{\sum_{T \in \mathcal{T}\left(\check{Z}_{N_{p}}^{(p)}\right)} \prod_{T_{i} \in T} \beta_{p}\left[T_{i}, 1\right]} \\
\left.\left.\left(x \in T_{t}, \delta_{x}\right] \prod_{\substack{T_{j} \in T \\
T_{j} \neq T_{i}}} \beta_{p}\left[T_{j}, 1\right]\right), p \neq 0\right)
\end{array}\right.
$$

\footnotetext{
${ }^{5}\left\{P_{S}(p)\right\}_{p=1}^{P}=\left\{P_{S, k+1}(p)\right\}_{p=1}^{P_{k+1}}$ and $\left\{P_{T}(p)\right\}_{p=0}^{P}=$ $\left\{P_{T, k+1}(p)\right\}_{p=0}^{P_{k+1}}$, time subscripts are omitted for simplicity's sake.
}

where $\beta_{p}$ is the cross-term restricted to sensors $j \in P_{S}(p) \subset[1 \mathrm{~N}]$ and to the subregion $P_{T}(p) \subset \mathcal{X}, \mathcal{T}\left(\check{Z}_{N_{p}}^{(p)}\right)$ is the set of combinational terms restricted to measurements from sensors $j \in P_{S}(p)$. As illustrated on a simple scenario in [3], the "brute force" (5) and the partition method (9) both yield the true data updated density since (5) and (9) are equivalent, yet the partition method spares itself the computation of vanishing cross-terms and is therefore significantly lighter.

\section{SIMULATION}

Since the single-sensor equivalent of equation (4) has a nice analytical expression and is easy to compute ([2]), Mahler introduced the classical iterated-corrector approximation in which the singlesensor data update equation is applied $N$ times successively, considering the measurements from sensor $i$ at the $i$-th iteration. That is, the "iterated" method proceeds with sensors sequentially rather than in a whole. However, to the authors' knowledge, the quality of the approximation provided by the iterated method is unknown since it lacks a comparison with a true reference. The objective of this simulation is to evaluate the iterated method on a given scenario by comparing the PHD updated through the iterated method with the true reference PHD, available thanks to the partition method.

\subsection{Scenario description}

A target state $\mathbf{x} \in \mathbb{R}^{4}$ is composed of position $(x, y)$ and velocity $(\dot{x}, \dot{y})$ variables. Targets evolve according to a nearly constant velocity (NCV) model. The birth process is Poisson with a constant rate, new targets are spread uniformly in the state space. Targets die whenever reaching the edges of the 2-D position subspace. The test scenario lasts 500 time steps and involves up to 12 simultaneous targets. The ten sensors provide measurements with an independant Gaussian noise on range, bearing and eventually radial velocity. False alarms are Poisson and uniformly spread inside the FOV. Each sensor has its own set of sensing parameters (detection probability, FOV shape, false alarm rate, noise variances). Their FOVs are assumed fixed and spread as follows:

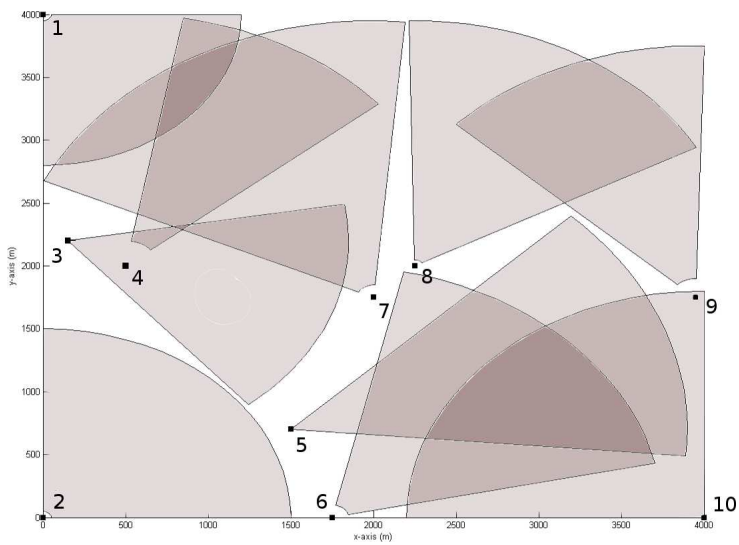

Fig. 1: Sensors' positions (dots) and FOVs in position subspace

The PHD multi-target tracker was implemented with a particle filter ([6]), in this particular case the "cross" relation (7) is restricted as follows: two sensors $j_{1}, j_{2}$ are said to cross each other if and only if at least one particle $\tilde{\mathbf{x}}^{i}$ belongs to both FOVs. Note (fig. 1) that the FOV configuration is such that the sensor partition at any time is a 
subdivision of the coarse partition $\{1-3-4-7,2,5-6-10,8-9\}$. Thus, the computational gain of the partition method over the brute force approach is likely to be significant regardless of the particle spreading.

\subsection{Results}

The same scenario (i.e with identical target behavior) has been run 10 times, maintaining simultaneously a partition-based PHD and a iterated-based PHD. The two densities are compared through the estimated target number (fig. 2) and the OSPA distance [7] between the set of real targets and the sets of PHD-extracted estimated targets (fig. 3). These two figures show that, on this particular scenario, the iterated method has similar performances than the partition method; although it does not appear in this paper for lack of space, this is further illustrated by the almost identical trajectories in the $2 \mathrm{D}$ position subspace of the PHD-extracted tracks given by both methods.

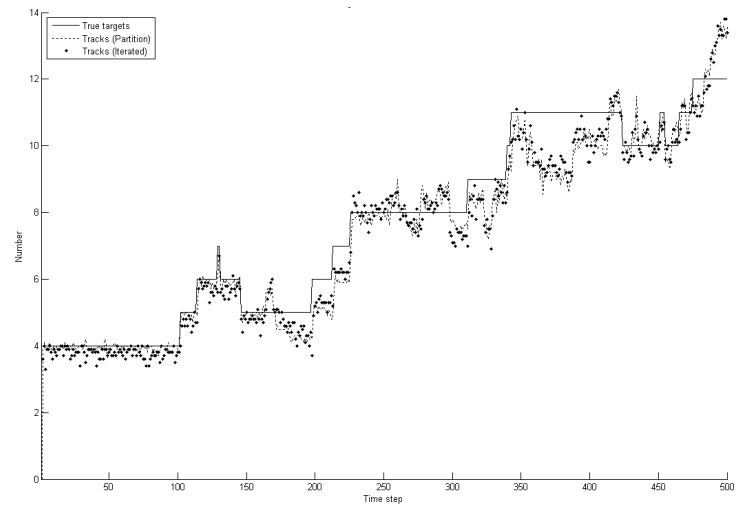

Fig. 2: Target number (true and estimated)

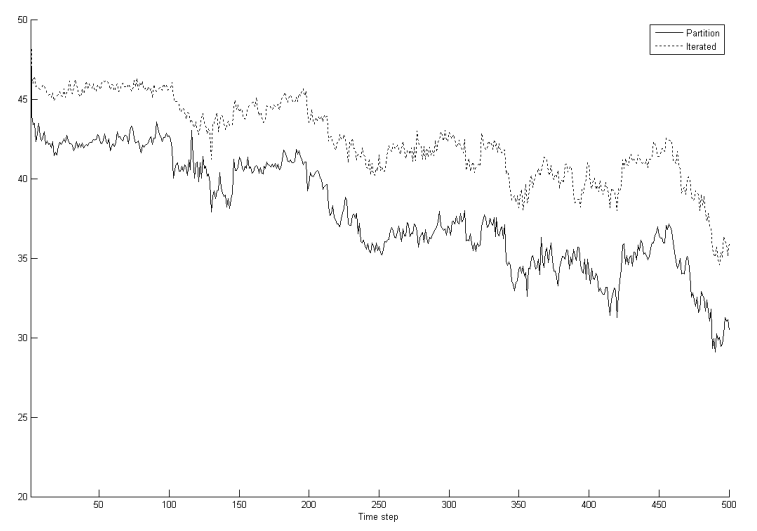

Fig. 3: OSPA distance $(\mathrm{p}=2, \mathrm{c}=50)$

Since the partition method is based on the propagation of the true PHD, this comparaison provides grounds for a validation of the iterated method within the PHD framework, albeit limited to this scenario and depending on the particle filter approximation. Note that this comparison could not have been properly implemented with the brute force approach: propagating the true density in this scenario without the partitionning proved to be too computationally demanding on a desktop computer using Matlab (with some embedded C code) whereas each run was performed in about two and a half minutes with the partition method.

\section{CONCLUSION AND FURTHER WORK}

In this paper, a new formulation of the partioning method for the practical implementation of an exact multi-sensor multi-target PHD filter has been proposed. Thanks to this method, the computational cost of the implementation of the multi-sensor multi-target data update step is significanlty reduced whenever the configuration of the sensor FOVs is favorable to a partitioning. This method is of practical interest because it allows the efficient computation of a reference density which is exact in the sense of Bayesian inference within the PHD framework. In this paper, the classical iterated-corrector approximation was compared to the exact multi-sensor PHD filter, available through the partition method, on a 10-sensor scenario. The results seem to indicate similar performances, for target number as well as target state estimation.

Furthermore, the partitioning method seems to offer new perspectives on the multi-sensor PHD problem. First, the same comparison could be implemented on various scenarios in order to validate the iterated approximation method on a broader range of situations involving different sensor and/or target behaviors. Then, since the partitioning shows that the data update step can be processed independently in each partition element of the state space, the authors believe that the approximation in the iterated-corrector method depends only on the order in which sensors are processed within their respective partition element rather than within all the sensors; should this result be verified, it could provide new leads for the resolution of the well-known sensor order issue in the various PHD iterative approximations. Finally, one may think of applying a similar partitioning technique in order to obtain a tractable PENT-based [8] multi-sensor manager; this is currently under consideration by the authors.

\section{REFERENCES}

[1] R. P. S. Mahler, Handbook of Multisensor Data Fusion, chapter 14, CRC Press, 2002.

[2] R. P. S. Mahler, "Multitarget bayes filtering via first-order multitarget moments," IEEE Transactions on Aerospace and Electronic Systems, vol. 39, no. 4, pp. 1152-1178, Oct. 2003.

[3] E. Delande, E. Duflos, D. Heurguier, and P. Vanheeghe, "Multisensor phd: construction and implementation by space partitioning," in Proceedings of the IEEE International Conference on Acoustics, Speech, and Signal Processing (ICASSP), May 2011, to appear.

[4] R. P. S. Mahler, "The multisensor phd filter, i: General solution via multitarget calculus," in Signal Processing, Sensor Fusion, and Target Recognition XVIII, Proceedings of SPIE, Apr. 2009.

[5] E. Delande, E. Duflos, D. Heurguier, and P. Vanheeghe, "Multitarget phd filtering: proposition of extensions to the multi-sensor case,” Research Report RR-7337, INRIA, July 2010.

[6] B-N. Vo, S. Singh, and A. Doucet, "Sequential monte carlo methods for multi-target filtering with random finite sets," IEEE Transactions on Aerospace and Electronic Systems, vol. 41, no. 4, pp. 1224-1245, Oct. 2005.

[7] B-T. Vo, Random Finite Sets in Multi-Object Filtering, Ph.D. thesis, The University of Western Australia, Oct. 2008.

[8] R. P. S. Mahler, "Sensor management with non-ideal sensor dynamics," in Information Fusion, 2004, Proceedings of the 7th International Conference on, June 2004. 